\title{
Focal segmental glomerulosclerosis: challenges in definitions, pathogenesis and management
}

\author{
John Feehally \\ University of Leicester, UK.
}

\section{ABSTRACT}

Focal segmental glomerulosclerosis (FSGS) is a well-defined pattern of glomerular injury identifiable on renal biopsy using light microscopy. FSGS is not a single entity and much information is needed to make a proper evaluation in each subject with the condition to identify the cause, prognosticate, and inform treatment choices. Categories of information required include: clinical presentation, responsiveness to steroids, pathological subtype, genetic background, and evidence for other adaptive, viral, and toxic causes. Primary FSGS describes a cohort of conditions identified by exclusion of known contributory causes, but does not represent a single entity. Clinical manifestations and outcomes of FSGS vary widely; they include asymptomatic proteinuria, cases of spontaneous remission, steroidsensitive nephrotic syndrome, and nephrotic syndrome resistant to immune modulating therapy progressing to end-stage renal disease with recurrence after transplantation. Although immune modulating therapy (based notably on corticosteroids and calcineurin inhibitors) are widely used in primary FSGS, robust evidence of their efficacy remains scant.

Keywords: FSGS review; proteinuria; nephrotic syndrome; immunosuppression.

\section{INTRODUCTION}

The term focal segmental glomerulosclerosis (FSGS) describes a characteristic pattern of glomerular injury which can be identified on light-microscopic (LM) examination of renal biopsy material. Glomeruli show segmental areas of sclerosis and hyalinosis which may progress to global glomerulosclerosis; in progressive cases there will also be increasing tubular atrophy and interstitial fibrosis. The FSGS pattern was identified by light microscopy soon after the introduction of renal biopsy into clinical practice in the 1950s. The introduction of immunofluorescence (and later immunoperoxidase) confirmed the absence of immune deposits (other than passive trapping of IgM sometimes seen within sclerotic areas). Electron microscopy is unavailable in many parts of the world; it is not mandatory for diagnosis but can add valuable information: by excluding small immune complexes and early amyloid deposition not visible on LM or immunofluorescence, by showing glomerular basement membrane changes resulting from collagen gene mutations which are occasionally associated with the FSGS pattern, and by providing an additional estimation of the extent of podocyte injury.

FSGS was originally associated with nephrotic syndrome in children and young adults, in patients who often had clinical presentations indistinguishable from those seen with minimal change disease (MCD): sudden onset of nephrotic syndrome, only manifesting haematuria or hypertension in a minority of cases, and with preserved glomerular filtration rate (GFR), unless there was marked volume depletion associated with the nephrosis. But it soon became clear that the FSGS pattern also occurs in a far broader range of settings. The condition has been increasing in prevalence in all parts of the world where there is reliable reporting. In some countries, for example Australia, this may reflect a liberal biopsy policy which offers biopsy to individuals with asymptomatic proteinuria as well as those with nephrotic syndrome [I]. But 
sequential data from Nigeria [2] and United States [3] show an increase in the incidence of FSGS; the increase in America is especially in those of African origin.

There has been much progress in our understanding of the aetiology and pathogenesis of FSGS, and its recognition as a consequence of primary podocyte injury, and new treatments are gradually emerging. However, there remain a number of issues where clarity is still lacking, and which can cause confusion. In this article I discuss some of these issues, which include matters of definition and terminology, classification, prognostication, and choice of therapy.

\section{DEFINITIONS AND TERMINOLOGY}

The terms FSGS and MCD have clear and precise definitions of their pathology. But the apparently close relationship between FSGS and MCD leaves unresolved the questions of whether the two are separate entities, or whether MCD can evolve into FSGS. Some pathologists therefore favour the inclusive term 'MCD-FSGS spectrum'. The 'tip lesion' and 'collapsing glomerulopathy' have also been defined histologically but controversy still persists about whether these are distinct entities or are variants of FSGS.

\section{Podocytopathy}

In an attempt to resolve this lack of clarity, the term 'podocytopathy' has been proposed [4], encompassing a variety of histological patterns thought to follow a common mechanism of primary injury to the podocyte, reflecting the rapid increase in our knowledge of podocyte pathobiology over the last 20 years. However, the term 'podocytopathy' has not brought the clarity that was hoped for. It has often been used loosely to include a wide range of podocyte injury, and indeed sometimes to imply only podocyte hypertrophy. Proponents of the term 'podocytopathy' use it to embrace MCD, FSGS, tip lesion, collapsing glomerulopathy, and diffuse mesangial sclerosis; but they do not include other conditions in which the podocyte injury is fundamental to the disease process - be it metabolic (as in diabetic nephropathy) or an auto-immune process (as in membranous nephropathy). I therefore do not favour 'podocytopathy' since it seems to add neither pathogenic, diagnostic nor therapeutic clarity.

\section{Idiopathic nephrotic syndrome}

Another term best avoided is 'idiopathic nephrotic syndrome' - which in the past was widely used to bring together MCD and FSGS in adults, and steroid-sensitive nephrotic syndrome (SSNS) and steroid-resistant nephrotic syndrome (SRNS) in children. Use of this term has diminished as the aetiology and pathogenesis of FSGS are becoming better understood.

\section{Steroid responsiveness in nephrotic syndrome}

The different clinical approaches to nephrotic syndrome in children and adults, while representing sensible pragmatic clinical practice, add uncertainty which complicates the classification of MCD and FSGS. The great majority of those presenting with nephrotic syndrome (adults or children), who have a biopsy showing MCD, will be steroidsensitive, that is, will have a complete remission of proteinuria in response to corticosteroid therapy. It has been known, from biopsy studies in children with nephrotic syndrome by the International Study of Kidney Disease in Children (ISKDC), that the great majority of nephrotic children have MCD. This has led to the routine use of corticosteroids without renal biopsy for children presenting with 'typical' nephrotic syndrome, who will mostly prove to have SSNS. However, the ISKDC studies showed that not all children with MCD are steroid-sensitive. Do such cases truly have MCD or is there another histological pattern (most commonly FSGS), which would be detected with more thorough biopsy analysis? On the other hand, not all children with SSNS have MCD, some will have FSGS. Resistance to initial steroid therapy defines steroid-resistant nephrotic syndrome in children, but this is not synonymous with FSGS, since there may be other histological patterns in this setting. Although clinical practice guidelines (including the KDIGO guideline [5]) recommend that all children with SRNS should undergo renal biopsy, this is not always done in routine clinical practice, which perpetuates the uncertainty about the proportion of those with SRNS who have FSGS. In adult practice, initial renal biopsy remains the standard approach so that the histological pattern is known when therapy is started. These differences in practice, and between adults and children, and the incomplete correlation between steroid sensitivity and histological pattern, have added to our lack of clarity about definitions, and present challenges when, for example, extrapolating guidance on treatment choices for FSGS in adults from data on SRNS in children.

\section{FSGS as pattern not disease}

The FSGS pattern seen on biopsy cannot be attributed to a single disease process and may be the consequence of a wide range of glomerular insults (Figure I). These include viral infections, medications and toxins, and an adaptive response to inherited or acquired reduction in glomerular number, genetic susceptibility genes, and genetic mutations in podocyte proteins. Terminology is in flux, making the older literature hard to interpret. Most use the term 'secondary' FSGS when one or more of these underlying causes shown in Figure $I$ is identified. 'Primary' FSGS is typically used to mean those in whom such secondary causes are not found. Others prefer the term 'idiopathic' rather than 'primary' FSGS. 


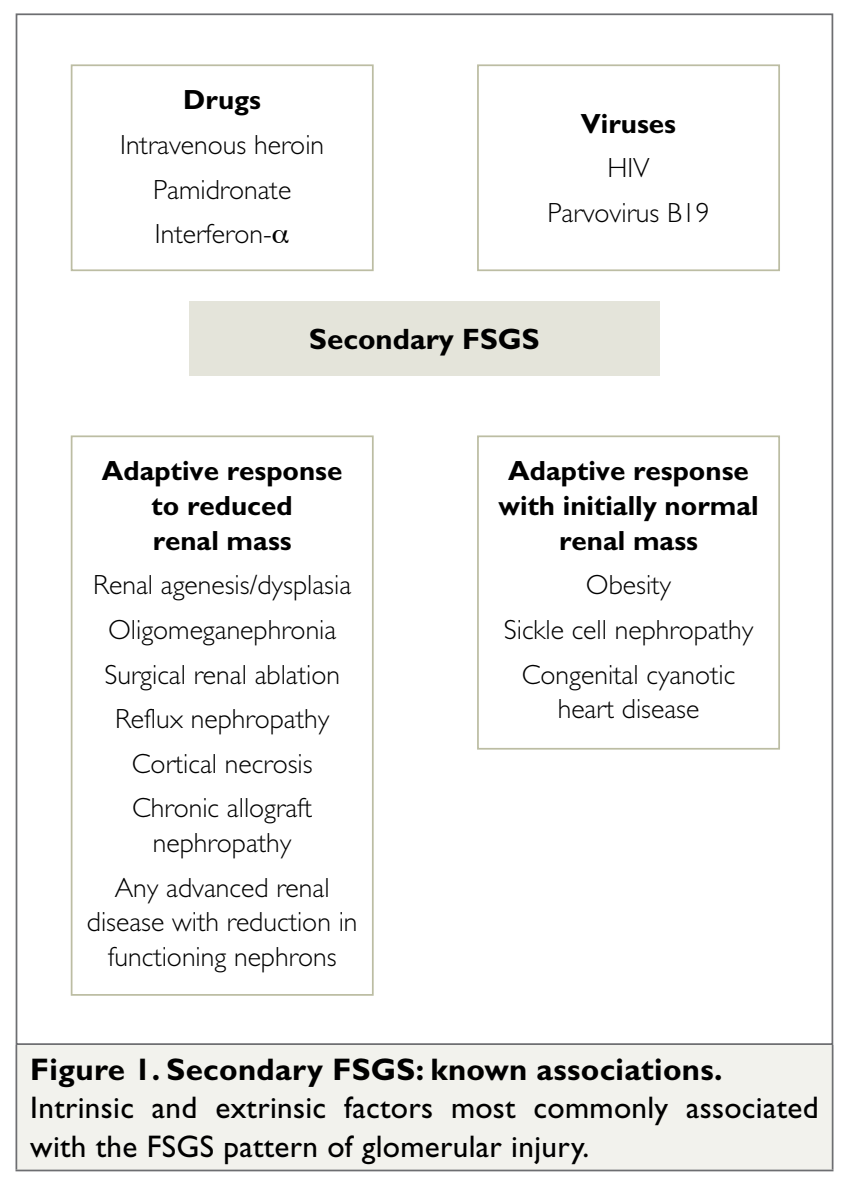

Clinical and pathological features may be suggestive in distinguishing primary from secondary FSGS. Frank nephrotic syndrome is more common in primary FSGS, in which foot process effacement is typically seen in at least $80 \%$ of glomerular capillary loops visualised on electron microscopy. But these are no more than pointers, and no substitute for thorough clinical and laboratory evaluation to look for underlying causes.

\section{'Primary' FSGS}

'Primary' FSGS is not a single entity. Some cases seem closely related to MCD and are steroid-sensitive; some may have a circulating factor which is thought to be the primary pathogenic mechanism; susceptibility to FSGS and its progression may be driven in some by their genetic background (see below). More than one of these mechanisms may be present in each case.

The clinical spectrum of primary FSGS includes the following: nephrotic syndrome which is fully steroidsensitive and never relapses; nephrotic syndrome which remits and relapses spontaneously; the clinical entity sometimes called 'malignant FSGS' [6] which is resistant to all immune modulating therapy, causes renal failure in months, and relapses immediately in the renal allograft, causing early graft failure. Such striking variety in clinical patterns argues against a single dominant pathogenic mechanism in primary FSGS.

\section{Pathological variants of FSGS}

Early work on FSGS described a single characteristic morphology. But subsequently other patterns have been recognised, and their relationship to FSGS continues to be debated.

\section{Tip lesion}

In the tip lesion, first described in 1984 [7], there is podocyte prominence and capsular adhesion adjacent to the urinary pole, with no other segmental sclerotic lesions. Nephrotic syndrome is characteristic, with a high chance this will be steroid sensitive. Is the tip lesion a variant of MCD or a variant of FSGS? Is it a cause or a consequence of heavy proteinuria? Given the lack of other glomerular injury, and the high rate of steroid sensitivity, some have regarded it as a variant of MCD, although it is more commonly described as a variant of FSGS. Indirect evidence that the tip lesion may be a consequence rather than a cause of heavy proteinuria comes from a unique study of kidneys from autopsies performed before 1950 in children and adults who died of infection with 'lipoid nephrosis' (an early term encompassing MCD and FSGS). This was in the pre-corticosteroid and pre-antibiotic era, so these subjects had prolonged nephrotic syndrome. No case had pathological features of FSGS, but the majority had tip lesions [8].

\section{Collapsing glomerulopathy}

The characteristic morphology of collapsing glomerulopathy was first described in 1986 [9], and includes glomerular tuft collapse with widespread podocyte hypertrophy and hyperplasia. Although strongly associated with HIV infection in early reports, it also occurs in the absence of viral infection or any other apparent cause, and is more common in people of African origin. On morphological grounds collapsing glomerulopathy is usually considered a variant of FSGS, though some regard it as a separate entity.

\section{Other pathological variants of FSGS}

The most widely used classification of pathological features in FSGS includes the tip lesion and the collapsing variant. It also identifies perihilar and cellular variants of FSGS, describing all other cases as FSGS NOS (not otherwise specified) [10].

Primary FSGS can be associated with FSGS NOS, or tip lesion, or the collapsing variant. It has been suggested that the perihilar variant may be more commonly seen in secondary adaptive FSGS, but uncertainty remains about the clinical utility of identifying the perihilar and cellular variants, not least because they are uncommon, comprising together only $10 \%$ of cases from a clinical trial in children and young adults with FSGS [II]. 


\section{GENETIC FACTORS IN FSGS}

The importance of genetic susceptibility to FSGS has received increasing recognition over recent years. First, there are associations with variants in susceptibility genes, which increases the risk of developing FSGS. The most common example is the APOLI gene in those of subSaharan African descent. One third of FSGS in the United States is associated with APOL I variants [12]. This is usually recessive, requiring two risk alleles, although a report from South Africa indicates that a single APOLI allele is associated with HIV-associated nephropathy [13]. Specific new therapies may emerge from the current intensive study of the molecular pathways of the APOLI variant involved in disease risk.

APOLI is a susceptibility gene, that is, most patients carrying two risk alleles do not get FSGS. This contrasts with the other type of genetic FSGS associated with high penetrance mutations with Mendelian inheritance. Some 40 mutations have now been reported affecting podocyte proteins (summarised in [14]), and it is highly likely more will emerge, as genetic techniques advance. The mutations have characteristic age distributions, some strongly associated with congenital or very early onset nephrotic syndrome, others with clinical onset in later childhood or adulthood [15].

Knowledge of these mutations has mostly emerged from the study cohorts including children and young adults with SRNS, some of whom have patterns of glomerular injury other than FSGS. Many cohorts are also enriched for those with a known family history of proteinuria or nephrotic syndrome, so there is less certainty about the prevalence of mutations in those with apparently sporadic FSGS. Nor indeed is the prevalence of these mutations known in SSNS or in MCD in adults, since these populations have not been systematically studied. Available data suggest that a mutation may be found in up to $60 \%$ of children presenting with SRNS in the first year of life, falling to 10\% in the second decade of life, and most often in those with a known family history [15].

There is a debate about the role of mutation testing in the evaluation of FSGS in routine clinical care, especially since such testing is unavailable or unaffordable in many parts of the world - although costs are declining as limited gene panels tailored for specific age groups become available. Testing in adults can be restricted to those with a family history of nephrotic syndrome in whom a mutation is not yet identified in another family member. Some argue that all children with SRNS should be tested because there 5 I may be implications for therapy or risk of transplant recur- rence. A more prevalent view is that testing should only be offered for children presenting in the first year of life or those with a family history.

\section{THE RELATIONSHIP BETWEEN MCD AND FSGS, AND BETWEEN PATHOLOGICAL PATTERN AND STEROID SENSITIVITY}

\section{MCD and FSGS}

When a case of nephrotic syndrome with a biopsy apparently showing MCD proves resistant to corticosteroids, the suspicion of unidentified FSGS is raised, and a more thorough review of the biopsy or even a repeat biopsy is recommended. This is because the focal nature of the FSGS lesion, and its initial predilection for deep juxtamedullary glomeruli, increase the likelihood that the lesions may have been missed especially in small or superficial biopsy samples. On the other hand, if an individual with nephrotic syndrome and MCD responds promptly to corticosteroids, the conventional presumption is that the diagnosis is MCD, and the biopsy will not be reviewed with such additional rigour, making it uncertain what proportion of those with steroid-sensitive 'MCD' do indeed have FSGS. If a second renal biopsy shows FSGS when the first did not, it may be that FSGS was missed in the first biopsy but an alternative explanation is that MCD can evolve into FSGS with time. This has some support from observations in post-transplant recurrence of FSGS, where onset of proteinuria may be almost immediate after transplantation. Moreover, a biopsy at this stage may show ultrastructural evidence of foot process effacement but segmental sclerotic lesions are absent and may not appear until weeks or months after clinical recurrence.

\section{Pathological pattern and steroid sensitivity}

The steroid sensitivity of nephrotic syndrome does not map neatly to pathological findings. Not every subject with biopsy findings of MCD is steroid-sensitive (though steroid-resistant cases might have FSGS which remains unidentified because of sampling issues in the biopsy). But some cases of FSGS are steroid-sensitive, and subsequently behave like MCD, a proportion having multiple relapses. Cases of FSGS in which the post-transplant clinical course strongly implicates a causative circulating factor are, by definition, steroid-resistant since they have developed end-stage renal disease (ESRD) despite all therapeutic efforts. There are no data to indicate whether some or all of those who have steroid-sensitive FSGS have a causative circulating factor. 
Initial steroid sensitivity is the single most powerful feature in predicting good long-term outcome in FSGS. From the patient's point of view this is what matters, and is much more important than the details of the renal biopsy findings, which tend to preoccupy nephrologists. Indeed, it could be argued that steroid sensitivity should define disease categories (as has happened for practical operational reasons in children) rather than the biopsy findings.

\section{DOES PRIMARY FSGS HAVE A SINGLE UNIQUE PATHOGENESIS?}

One justification for the term 'podocytopathy' was that it brings together a group of disorders with common pathogenesis, even though histological features may vary. Thus, it is argued that MCD, tip lesion, FSGS NOS, and collapsing glomerulopathy can be considered part of a spectrum with genetic factors and glomerular size - itself in part genetically patterned - influencing the eventual morphological and functional manifestations of the disease. Others propose that MCD and FSGS have separate pathogenesis, but because the podocyte has a limited repertoire of responses to injury, they share morphological evidence of widespread podocyte injury and the clinical features of nephrotic syndrome. Apart from the intellectual challenge of resolving this longstanding conundrum, the implications for strategies to develop new therapies are important. Should a single therapy be sought for a single disease mechanism? Or should therapeutic development focus separately on MCD and FSGS?

There is inconsistent evidence that MCD and FSGS each has unique a podocyte pathobiology. CD80 (B7-I) has been one focus of study. An initial report suggested that raised urinary $\mathrm{CD} 80$ was found in $\mathrm{MCD}$ only in relapse [16]. A report of increased podocyte CD80 (B7-I) expression in recurrent FSGS prompted the use of abatacept (an anti-B7-I agent) with anecdotal success in treating recurrent FSGS [17]. However, neither the podocyte expression variations nor the response to abatacept has proved robust in further studies [18].

The notion which has received sustained interest is that primary FSGS is caused - at least in some cases - by a small molecular weight circulating factor. Suggestive clinical evidence comes from the immediate recurrence of FSGS after transplantation in some subjects, and the prompt response of recurrence in many such cases to plasma exchange. This is supported by a striking case report of a kidney transplanted into a patient with FSGS with immediate recurrence and biopsy evidence of foot process effacement; the kidney was removed and transplanted into another recipient without FSGS where it functioned
The failure to identify such a circulating factor unequivocally despite intensive study over more than 20 years has been a disappointment. Among recent candidates (reviewed in [20]) the most promising was soluble plasminogen activating receptor (suPAR) which was initially reported to associate strongly with FSGS, but not other glomerular diseases. However, more recent studies do not confirm its specificity, and suggest that circulating suPAR levels are correlated with GFR [2I]. The lack of a measurable circulating factor means that its possible role in the pathogenesis of FSGS outside the specific posttransplant environment is unknown. Thus, it remains unknown what proportion of those with FSGS who are steroid-sensitive, or who are steroid-resistant but do not progress to ESRD, have a causative circulating factor.

\section{MAKING A COMPLETE DIAGNOSIS IN FSGS}

Physicians value classification of disease because it provides a framework which is expected to bring clarity and accuracy - with positive impacts on precise diagnosis, accurate prognosis, and personalised therapy. An informative and accurate classification of FSGS has, however, proved elusive because of the complexity of factors which must be considered.

Pathological findings have dominated thinking about the classification of glomerular disease since the introduction of renal biopsy in the 1950s. The pathology was soon taken to define each 'disease'. For example both FSGS and membranous nephropathy were typically described as 'diseases' even though insights into their aetiology and pathogenesis were scanty. An appropriate retreat from this position now emphasises that pathological findings represent 'patterns' not 'diseases', and identification of such a pattern is the starting point for further evaluation and investigation to clarify the diagnosis as precisely as possible.

Current attempts to classify FSGS typically tabulate the factors now known to cause FSGS (Figure I), and recommend that each case be assigned to one cause - for example, adaptive changes to low nephron number, viral infection, medications and other toxins, APOLI polymorphisms, or podocyte protein mutations. These various entities are together usually called 'secondary' FSGS. Such 'classification' has two important limitations. First, it creates an unsatisfactory category, 'primary' FSGS, which is merely a diagnosis by exclusion and is not in any sense a single clinicopathological entity. Despite our preference for tidy classification, our current understanding of FSGS does not allow us to assign cases neatly in this way. For each case, at least five domains should be considered and described, each of which influences our understanding of 


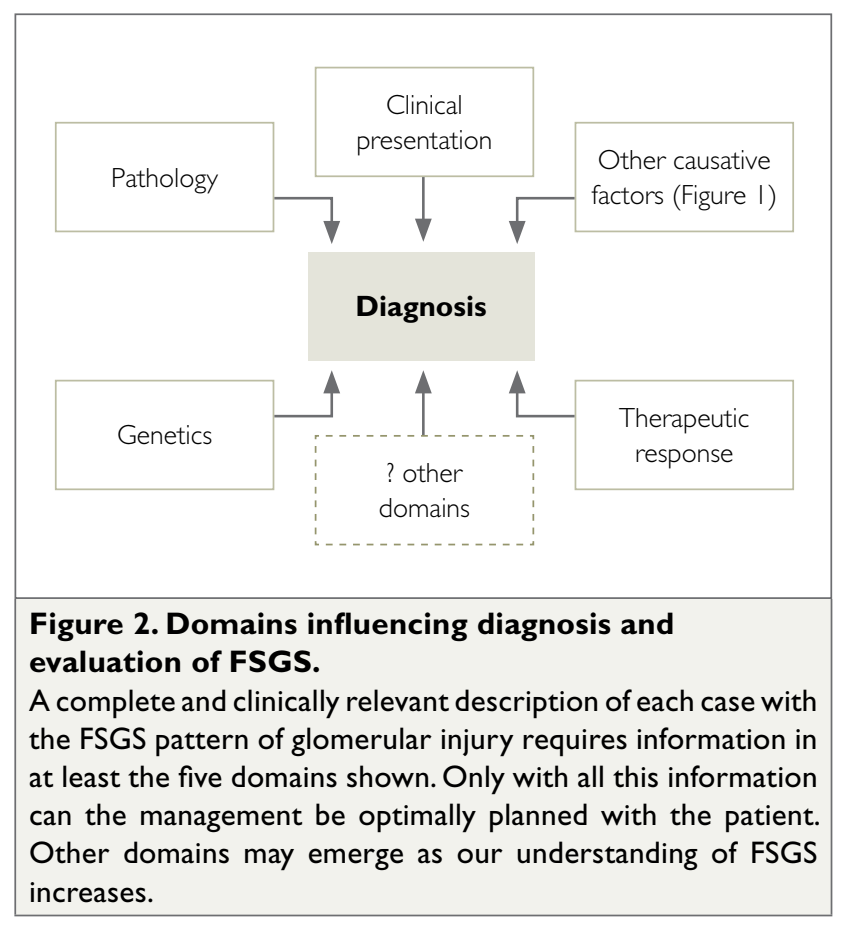

the case and its clinical management. The five domains are: clinical presentation, pathological subtype, genetic background, other causative 'secondary' features, and steroid sensitivity (Figure 2).

The statement "This patient with FSGS ..." is often heard. Nephrologists revert consciously or unconsciously to the misunderstanding that it is the pathological pattern which defines the disease. While it may be acceptable shorthand, it can also betray limitations in understanding the multiple domains of any particular case, and indeed of FSGS in general. In part, this taxonomic difficulty is understandable since it is very difficult conceptually to conflate the five domains to express fully the overall understanding of a case. It is especially challenging for nephrologists and pathologists to synthesise a case and discuss with each other when analysis in five dimensions is required. It is even more challenging to explain adequately to a patient with FSGS and their carers, referring to all five dimensions, the complexity of the situation which confronts them.

\section{PREDICTING PROGNOSIS IN FSGS}

Reliable prediction of prognosis is an important goal for any condition in order for the patient and their family to understand the implications of the diagnosis and to plan for the future, including working with their physician to agree on the appropriate therapy. Predicting prognosis becomes even more important when the range of possible outcomes and their implications are as broad as in FSGS. The possible outcomes include, on the one hand, a spontaneous remission or a complete response to corticosteroids with no relapse; and on the other hand, rapid progression to ESRD, and early recurrence after transplant, leaving longterm dialysis therapy as the only life-sustaining option.

\section{Clinical features}

Complete remission of nephrotic syndrome is the single most powerful predictor of good prognosis, but it should not be forgotten that partial remission - variably defined as reduction in proteinuria to below the nephrotic range, or as halving of the initial proteinuria - also provides significant protection against risk of ESRD [22].

For a subject with FSGS who has reduced GFR at presentation - assuming that it is not due to volume depletion or some other reversible consequence of nephrotic syndrome - the die is cast, and progression to ESRD is highly likely even though skilful care may delay the outcome.

\section{Pathological features}

Efforts to use pathological features to assist in prediction of outcome have met with mixed success. Most studies show that the collapsing variant carries an overall worse prognosis, and the tip lesion a better prognosis than 'typical' FSGS. The cellular and perihilar variants have not been shown to have prognostic value, although in most series these two patterns are too infrequent to exclude their relevance with certainty $[\mathrm{I} \mid]$. The possibility of sampling error in a focal entity such as FSGS means that caution should be exercised in using pathological features, such as tubular atrophy and interstitial fibrosis or proportion of sclerotic glomeruli, to predict risk and rate of progression.

\section{CHOOSING TREATMENT FOR FSGS}

'Supportive care' is the term often used for management of proteinuric kidney disease with approaches other than immunomodulatory therapy. This is applicable to all cases with FSGS regardless of the underlying aetiology and pathogenesis. The goals of supportive care include tight blood pressure control, minimisation of proteinuria and reduction of concomitant cardiovascular risk as well as specific complications of nephrotic syndrome. 'Supportive care' includes use of renin-angiotensin system (RAS) blockade and other anti-hypertensives with dietary salt restriction to maximise their efficacy, diuretics and statins. Whether RAS blockade should include ACE inhibitors and angiotensin receptor blockers in combination, and the possible roles of direct renin inhibitors and aldosterone antagonists, are important clinical questions, outside the scope of this article. Here I focus on possible therapeutic roles for immune modulating agents - particularly corticosteroids, calcineurin inhibitors, and mycophenolate. 
Precise diagnosis as emphasised above naturally forms the basis for rational choice of therapy. Systematic evidence is limited to studies in those with nephrotic syndrome; there are almost no data to support a role for immunomodulatory therapy in FSGS with subnephrotic proteinuria. Most available evidence on immunosuppressive therapy comes from studies in those presumed to have primary FSGS.

The best available summary of evidence for therapy in FSGS is published in the KDIGO clinical practice guideline for glomerulonephritis [5]. The guideline summarises all studies published up to $201 \mathrm{l}$, and is presently being updated. In the KDIGO guideline, separate chapters summarise the evidence for treatment of MCD and FSGS in adults, and for SSNS and SRNS in children. The evidence base for treatment is significantly greater in children; there is uncertainty about direct extrapolation between SSNS and SRNS in children, and MCD and FSGS, respectively, in adults.

\section{Corticosteroids}

Since the 1950s, corticosteroids have been widely used in adults with FSGS. The rationale for their use was never clear, other than the observation that the majority of those with MCD are steroid-sensitive, and the close relationship between MCD and FSGS. It remains unknown, 67 years after the first reports of the efficacy of corticosteroids in childhood nephrotic syndrome (23), why this therapy is effective.

There are no RCTs of corticosteroids in FSGS in adults. Evidence comes from retrospective cohort studies of adults with FSGS and nephrotic syndrome in whom no underlying cause was identified and who are therefore regarded as having primary FSGS. Initially, these studies were from the 1980's, mostly using oral prednisolone typically at $1 \mathrm{mg} / \mathrm{kg} /$ day for 8 weeks (based on a typical regimen used for SSNS in children). Such studies typically report complete remission rates of 20-30\%. In the 1990s longer durations and higher doses came into favour, notably in the United States; up to 4 months of oral prednisolone at an initial dose of $\mathrm{I}-2 \mathrm{mg} / \mathrm{kg}$ was common, and complete remission rates of 50-60\% were reported [24]. Current clinical practice guidelines (including KDIGO) recommend this higher dose therapy as immediate initial therapy in those presenting with nephrotic syndrome and primary FSGS, although the length of time the starting dose is maintained before tapering has remained variable.

However, the weaknesses of retrospective observational studies as a basis for deciding therapy should not be old. Was secondary FSGS excluded with the rigour expected in contemporary clinical practice? Were histological features defined with the thoroughness used today? Were the genetics of the cases defined? Was 'supportive care' including RAS blockade optimised? Inevitably the answer to all these questions in reports from that era will be 'no'. Studies are also confounded by variable definitions of complete, and especially of partial, remission; and also by uncertainties about inclusion criteria. Were all consecutive cases included? What criteria were used to select patients who were not given corticosteroids? Nor is there systematic and thorough evaluation of both early and late complications of prolonged high dose corticosteroid therapy in the early observational studies, limiting the opportunity for adequate analysis of risk against benefit.

Another critical issue in justifying the need for prolonged corticosteroid therapy is the rate of spontaneous remission of nephrotic syndrome in primary FSGS. A substantial spontaneous remission rate might cause the treatment strategy to be modified. For example, in the absence of severe morbid nephrotic syndrome or severe nephrotic complications, it might be appropriate to observe progress before deciding whether corticosteroid or other immune modulating therapy is indeed necessary.

Studies from the United States have shown spontaneous remission rates of 4-6\%, whereas one European study reported a 16\% spontaneous remission (25), and a UK study reported 23\% [26]. If these higher spontaneous remission rates were indeed confirmed, this would shift the balance of risk and benefit in deciding if corticosteroids are required. A challenge is that the early use of prolonged courses of corticosteroids is so widespread that it is now very difficult to establish spontaneous remission rates in contemporary practice, or to tell whether resolution of nephrotic syndrome after four months of corticosteroids is indeed a response to a therapy or a spontaneous remission.

\section{Predicting steroid resistance}

More selective use of corticosteroids depends on the extent to which other features of each case can be used to predict steroid resistance. Thus, steroid resistance can be expected in those with FSGS associated with podocyte mutations. This might not always be the case, however, in those who present in the second or third decade of life, since there must be the possibility that a 'second hit' has allowed clinical expression to emerge, and that this second hit might be responsive to steroids. Ancestry is also relevant; studies from the US suggest that African Americans are less likely to be steroid-responsive. 
Whereas those with the tip lesion are commonly steroid sensitive, the collapsing variant has been regarded as typically steroid-resistant. However, a recent large retrospective study from the US reported a 70\% response to immunotherapy (corticosteroids or $\mathrm{CNI}$ ) in collapsing FSGS compared to $65 \%$ in those with FSGS NOS [27]. There were also much higher spontaneous remission rates than previously reported from the US: $40 \%$ in collapsing FSGS with nephrotic syndrome, and 56\% in FSGS NOS (however, these spontaneous remissions included some who were not nephrotic, and among those with nephroticrange proteinuria none had serum albumin <33 g/L). Although only a retrospective study, these recent data nevertheless support the case for a 'watch and wait' approach in FSGS with nephrotic syndrome (unless the patient has severe nephrotic syndrome with complications) as recommended for membranous nephropathy.

There are also preliminary reports that altered expression of glomerular proteins, including dystroglycan [28], as well as variations in glucocorticoid receptor polymorphisms [29], may have a role in predicting steroid responsiveness, but these require confirmation.

Regrettably, it seems very unlikely that an RCT of corticosteroids, in cohorts of FSGS patients carefully categorised in the light of current knowledge, which is needed to resolve properly these uncertainties, will ever be carried out.

\section{Calcineurin inhibitors}

A widely used alternative to corticosteroids is calcineurin inhibitors (CNI). Again, the rationale is not entirely clear; proteinuria reduction by $\mathrm{CNI}$ may be due to immunosuppressive or non-immunosuppressive effects, the latter including haemodynamic effects that reduce GFR and thus proteinuria, or direct podocyte effects.

RCTs of cyclosporine - sometimes in combination with low dose oral corticosteroids - show partial or complete remission rates of $70-75 \%$, although more than half may relapse after $\mathrm{CNI}$ withdrawal (summarised in [5]). A more recent RCT using tacrolimus showed similar efficacy [30].

\section{Mycophenolate}

Evidence assessing the role of mycophenolate in FSGS is limited. A small RCT comparing a mycophenolate-corticosteroid combination with cyclosporine in steroid-resistant FSGS showed no difference in remission rates or in the maintenance of remission in the six months after stopping therapy [3I]. Three other small RCTs evaluating mycophenolate combined with corticosteroids showed no benefit; non-randomised studies in steroid-resistant FSGS

\section{Other treatment options}

A range of agents directed at other possible mechanistic pathways in FSGS have been evaluated in observational studies, or are under investigation in RCTs, but none has supportive evidence sufficient to make their use anything other than speculative in current clinical practice.

\section{Conflict of interest}

None to declare.

\section{REFERENCES}

I. McGrogan A, Franssen CF, de Vries CS. The incidence of primary glomerulonephritis worldwide: a systematic review of the literature. Nephrol Dial Transplant. 20 I I; 26(2):4 I4-430.

2. Asinobi AO, Ademola AD, Okolo CA, Yaria JO. Trends in the histopathology of childhood nephrotic syndrome in Ibadan Nigeria: preponderance of idiopathic focal segmental glomerulosclerosis. BMC Nephrol. 2015; 16:213.

3. Kitiyakara C, Eggers P, Kopp JB. Twenty-one-year trend in ESRD due to focal segmental glomerulosclerosis in the United States. Am J Kidney Dis. 2004; 44(5):81 5-825.

4. Barisoni L, Schnaper HW, Kopp JB. A proposed taxonomy for the podocytopathies: a reassessment of the primary nephrotic diseases. Clin J Am Soc Nephrol. 2007; 2(3):529-542.

5. Kidney Disease: Improving Global Outcomes (KDIGO) Glomerulonephritis Work Group. KDIGO Clinical Practice Guideline for Glomerulonephritis. Kidney Int Suppl. 2012; 2:I 39-274.

6. Brown CB, Cameron JS, Turner DR, Chantler C, Ogg CS, Williams $D G$, et al. Focal segmental glomerulosclerosis with rapid decline in renal function ("malignant FSGS"). Clin Nephrol. 1978; I0(2):5 I-6I.

7. Howie AJ, Brewer DB. The glomerular tip lesion: a previously undescribed type of segmental glomerular abnormality. J Pathol. 1984; | 42(3):205-220.

8. Haas M, Yousefzadeh N. Glomerular tip lesion in minimal change nephropathy: a study of autopsies before 1950. Am J Kidney Dis. 2002; 39: | | 68-। | 75 .

9. Weiss MA, Daquioag E, Margolin EG, Pollak VE. Nephrotic syndrome, progressive irreversible renal failure, and glomerular "collapse": a new clinicopathologic entity? Am J Kidney Dis. 1986; $7(1): 20-28$.

10. D'Agati VD, Fogo AB, Bruijn JA, Jennette JC. Pathologic classification of focal segmental glomerulosclerosis: a working proposal. Am J Kidney Dis. 2004; 43(2):368-382.

I I. D'Agati VD, Alster JM, Jennette JC, Thomas DB, Pullman J, Savino DA, et al. Association of histologic variants in FSGS clinical trial with presenting features and outcomes. Clin J Am Soc Nephrol. 2013; 8(3):399-406.

12. Kopp JB, Nelson GW, Sampath K, Johnson RC, Genovese G, An P, et al. APOLI genetic variants in focal segmental glomerulosclerosis and HIV-associated nephropathy. J Am Soc Nephrol. 201 I; 22( I I):2129-2। 37 
13. Kasembeli AN, Duarte R, Ramsay M, Mosiane P, Dickens C, Dix-Peek $T$, et al. APOLI risk variants are strongly associated with HIV-associated nephropathy in Black South Africans. J Am Soc Nephrol. 20 I 5; 26( I I):2882-2890.

14. Rosenberg AZ, Kopp JB. Focal segmental glomerulosclerosis. Clin J Am Soc Nephrol. 2017; 12(3):502-517.

I5. Sadowski CE, Lovric S, Ashraf S, Pabst WL, Gee HY, Kohl S, et al. A single-gene cause in $29.5 \%$ of cases of steroid-resistant nephrotic syndrome. J Am Soc Nephrol. 20I 5; 26(6): I 279-I 289.

16. Garin EH, Diaz LN, Mu W, Wasserfall C, Araya C, Segal M, et al. Urinary CD80 excretion increases in idiopathic minimal-change disease. J Am Soc Nephrol. 2009; 20(2):260-266.

17. Yu CC, Fornoni A, Weins A, Hakroush S, Maiguel D, Sageshima J, et al. Abatacept in B7-I-positive proteinuric kidney disease. N Engl J Med. 2013; 369(25):2416-2423.

18. Salant DJ. Podocyte expression of B7-I/CD80: Is it a reliable biomarker for the treatment of proteinuric kidney diseases with abatacept? J Am Soc Nephrol. 20 I6; 27(4):963-965.

19. Gallon L, Leventhal J, Skaro A, Kanwar Y, Alvarado A. Resolution of recurrent focal segmental glomerulosclerosis after retransplantation. N Engl J Med. 2012; 366(17): I 648- 1649.

20. Königshausen E, Sellin L. Circulating permeability factors in primary focal segmental glomerulosclerosis: a review of proposed candidates. Biomed Res Int. 2016; $2016: 3765608$.

21. Meijers B, Maas RJ, Sprangers B, Claes K, Poesen R, Bammens B, et al. The soluble urokinase receptor is not a clinical marker for focal segmental glomerulosclerosis. Kidney Int. 2014; 85(3):636-640.

22. Troyanov S, Wall CA, Miller JA, Scholey JW, Cattran DC, Toronto Glomerulonephritis Registry Group. Focal and segmental glomerulosclerosis: definition and relevance of a partial remission. J Am Soc Nephrol. 2005; I6(4): 106 I- 1068.

23. Barnett HI, McNamara H, McCrory W, Forman C, Rapoport M, Michie A, Barbero G. The effects of ACTH and cortisone on the nephrotic syndrome. Am J Dis Child. 1950; 80(3):5 19-520.

24. Rydel JJ, Korbet SM, Borok RZ, Schwartz MM. Focal segmental glomerular sclerosis in adults: presentation, course, and response to treatment. Am J Kidney Dis. 1995; 25(4):534-542.

25. Ponticelli C, Rizzoni G, Edefonti A, Altieri P, Rivolta E, Rinaldi S, et al. A randomized trial of cyclosporine in steroid-resistant idiopathic nephrotic syndrome. Kidney Int. 1993; 43(6): I 377- I 384.

26. Stirling CM, Mathieson P, Boulton-Jones JM, Feehally J, Jayne D, Murray HM, Adu D. Treatment and outcome of adult patients with primary focal segmental glomerulosclerosis in five UK renal units. QJM. 2005; 98(6):443-449.

27. Laurin LP, Gasim AM, Derebail VK, McGregor JG, Kidd JM, Hogan SL, et al. Renal survival in patients with collapsing compared with not otherwise specified FSGS. Clin J Am Soc Nephrol. 2016; I I ( | 0): | 1752- 1759.

28. Giannico G, Yang H, Neilson EG, Fogo AB. Dystroglycan in the diagnosis of FSGS. Clin J Am Soc Nephrol. 2009; 4( I I): | 747- I 753.

29. Teeninga N, Kist-van Holthe JE, van den Akker EL, Kersten MC, Boersma E, Krabbe HG, et al. Genetic and in vivo determinants of glucocorticoid sensitivity in relation to clinical outcome of childhood nephrotic syndrome. Kidney Int. 20 | 4; 85(6): | 444- | 453.
30. Fan L, Liu Q, Liao Y, Li Z, Ji Y, Yang Z, et al. Tacrolimus is an alternative therapy option for the treatment of adult steroid-resistant nephrotic syndrome: a prospective, multicenter clinical trial. Int Urol Nephrol. 2013; 45(2):459-468.

31. Gipson DS, Trachtman H, Kaskel FJ, Greene TH, Radeva MK, Gassman JJ, et al. Clinical trial of focal segmental glomerulosclerosis in children and young adults. Kidney Int. 20I I; 80(8):868-878.

32. Beer A, Mayer G, Kronbichler A. Treatment strategies of adult primary focal segmental glomerulosclerosis: a systematic review focusing on the last two decades. Biomed Res Int. 2016; 2016: 4192578 . 